Mahmoud Y Taha

BVM\&S, MSc, PhD (Prof)

Nawal A AL-Sabawi

BDS, MSc (Assist Lect)

Enas Y Shehab

BSc, MSc (Assist Lect)

\section{Rapid Decontamination of Gutta Percha Cones Using Different Chemical Agents}

\author{
Department of Basic Dental Science
}

College of Dentistry, University of Mosul

Department of Conservative Dentistry

College of Dentistry, University of Mosul

Department of Basic Dental Science

College of Dentistry, University of Mosul

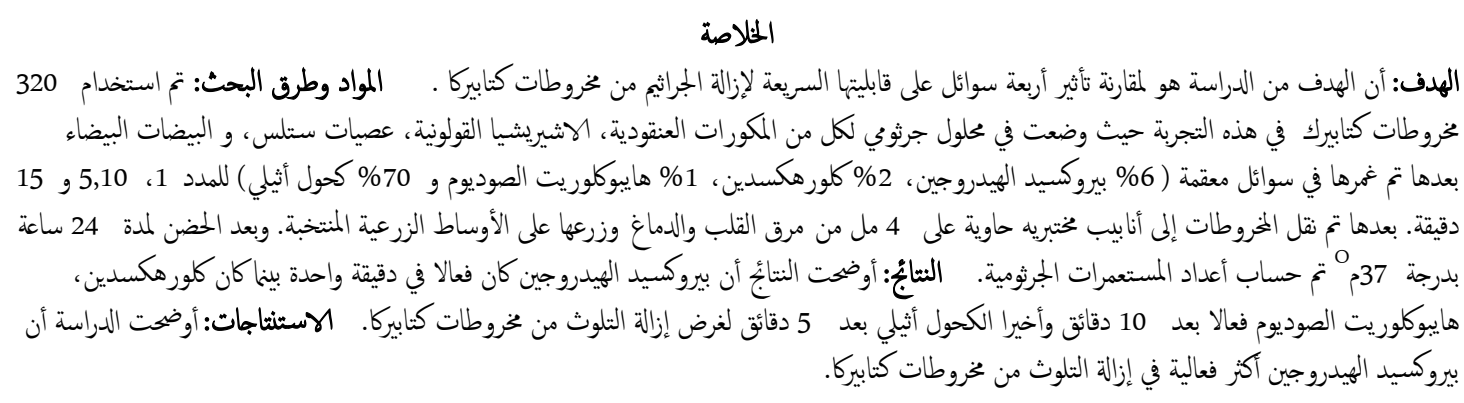

\title{
ABSTRACT
}

Aim: To compare the effectiveness of four different disinfectant solutions in rapid decontamination of gutta percha cones. Materials and Methods: In this experimental study, 320 gutta percha cones were placed in bacterial suspensions of Staphylococcus aureus, Escherichia coli, Bacillus subtilis, and Candida albicans for 30 minutes, and then immersed in disinfectant solutions (6\% hydrogen peroxide, $2 \%$ chlorhexidine, $1 \%$ sodium hypochlorite, and 70\% ethyl alcohol) for 1, 5, 10, and 15 minutes. After that, the cones were aseptically transferred to the test tubes containing $4 \mathrm{ml}$ brain heart infusion broth, and then cultured on selected agar media and number of colonies was estimated after 24 hours incubation at $37^{\circ} \mathrm{C}$. Results: Hydrogen peroxide was effective in 1 minute, while chlorhexidine and sodium hypochlorite after 10 minutes, and ethyl alcohol in 5 minutes for decontamination of gutta percha cones. Conclusion: Hydrogen peroxide was the most effective agents in decontamination of gutta perch cones.

Key Word: Decontamination, Gutta Percha, Chemical Agents.

Taha MY, Al-Sabawi NA, Shehab EY Rapid Decontamination of Gutta Percha Cones Using Different Chemical Agents. Al-Rafidain Dent J. 2010; 10(1):30-37.

Received:3/6/2008 Sent to Referees: $9 / 6 / 2008$

Accepted for Publication: 9/9/2008

\section{INTRODUCTION}

One of the primary objectives of root canal therapy is to eliminate or reduce microorganisms in the root canal. Decreasing the number of microorganisms without injuring adjacent vital tissues enhances endodontic success ${ }^{(1)}$. During endodontic therapy, an aseptic sequence is one of the professional main concerns and must not be broken ${ }^{(2,3)}$.

Care must be taken during root canal therapy to prevent contamination of filling materials and avoid root canal contamination. Gutta percha cones are now widely used to fill root canals. However they are not resistant to conventional sterilization processes in moist or dry heat. To keep the aseptic chain, gutta percha cones require rapid chair side decontamination before use $^{(4)}$

Literatures revealed several methods for rapid decontamination of gutta percha cones in dentistry. Among others, these include the following chemical agents: 
polyvinylpyrolidone iodine, glutaraldehyde, sodium hypochlorite, hydrogen peroxide, chlorhexidine, quaternary of ammonium, alcohol iodine, and ethyl alcohol. There is no agreement among national or foreign specialists for the best method $^{(5-8)}$.

The aim of this study was to evaluate the antimicrobial effect of different chemical agents used for decontamination of gutta percha cones contaminated with different microorganisms. The chemical agents used were $6 \%$ hydrogen peroxide, $2 \%$ chlorhexidine, $1 \%$ sodium hypochlorite, and $70 \%$ ethyl alcohol.

\section{MATERIALS AND METHODS}

In this experimental study, Gutta perch cones (ALPHA- DENT, INC, USA) size 80 was decontaminated by immersion in $6 \%$ sodium hypochlorite for 30 minute. The cones were individually and aseptically rinsed with sterile distilled water, and allowed to dry in Petri dishes containing sterile filter paper pads.

The disinfectant solutions used were $6 \%$ hydrogen peroxide, $2 \%$ chlorhexidine (Hibitane, 5\% (w/v), Zeneca Limited U.K), $1 \%$ sodium hypochlorite (FAS a commercial household bleach, 6.25\% (w/v), Iraq), and 70\% ethyl alcohol (Wadi Al-Rafidain Factory, Iraq).

Pure cultures of Staphylococcus aureus, Escherichia coli, Bacillus subtilis, and Candida albicans were obtained from Microbiology unit in the Department of Dental Basic Sciences. A bacterial suspension of 24 hours in brain heart infusion broth was used to contaminate of gutta percha cones by immersing them in $4 \mathrm{ml}$ of selected bacterial suspension for $30 \mathrm{mi}-$ nutes. The cones were transferred to sterile filter paper pads in Petri dish, and allowed to air dry for 5-10 minutes at room temperature under aseptic conditions. A series of 320 contaminated gutta percha cones was used. Five cones transferred individually to sterile tubes containing $10 \mathrm{ml}$ of the chemical agent to be tested and kept for $1,5,10$, and 15 minutes. Each cones was transferred to $4 \mathrm{ml}$ of brain heart infusion broth (Oxiod LTD, Basingstoke, Hants/ England). Gutta percha cones that decontaminated from Staphylococcus aureus and Bacillus subtilis were inoculated on brain heart infusion agar, those from Candida albicans on sabouraud agar (Oxiod LTD, Basingstoke, Hants/ England), and those from Escherichia coli on MacConky agar (Oxiod LTD, Basingstoke, Hants/ England), and incubated (Fisher Scientific/ Russia) for 24 hours at $37{ }^{\circ} \mathrm{C}$ and the number of colonies were estimated. Bacterial growth in inoculated plates indicated failure of decontamination and absence of growth indicated the efficiency of antimicrobial activity of the tested agents. For control of the experiments, 5 decontaminated cones (negative control), 5 contaminated cones (positive control) were individually cultivated in brain heart infusion broth, and another 5 contaminated cones immersed in distilled water for $1,5,10$, and 15 minutes were also cultured (washing control), all control group inoculated in selected agar media and incubated for 24 hours at $37^{\circ} \mathrm{C}^{(4,5,6)}$.

\section{RESULTS}

In this study, $6 \%$ hydrogen peroxide was the most effective agent because all gutta perch contaminated cones were decontaminated in 1 minute (Figure 1). Contaminated cones treated with $2 \%$ chlorhexidine showed decontamination effect on Staphylococcus aureus after 10 minutes and from Candida albicans after $5 \mathrm{mi}-$ nutes, while decontamination effect on Escherichia coli and Bacillus subtilis occurred in 1 minute (Figure 2).

Using ethyl alcohol at $70 \%$ showed that decontamination of cones from Staphylococcus aureus, Bacillus subtilis, and Candida albicans was achieved in 1 minute and from Escherichia coli after 5 minutes (Figure 3).

Finally, $1 \%$ sodium hypochlorite decontaminated the cones from Staphylococcus aureus, Bacillus subtilis, and Escherichia coli within 1 minute, but contaminated cones become sterilized from Candida albicans after 10 minutes as shown in Figure 4.

For control groups, negative control did not show bacterial growth, while positive control did, and washing control also revealed bacterial growth at all time intervals for all microorganisms. 


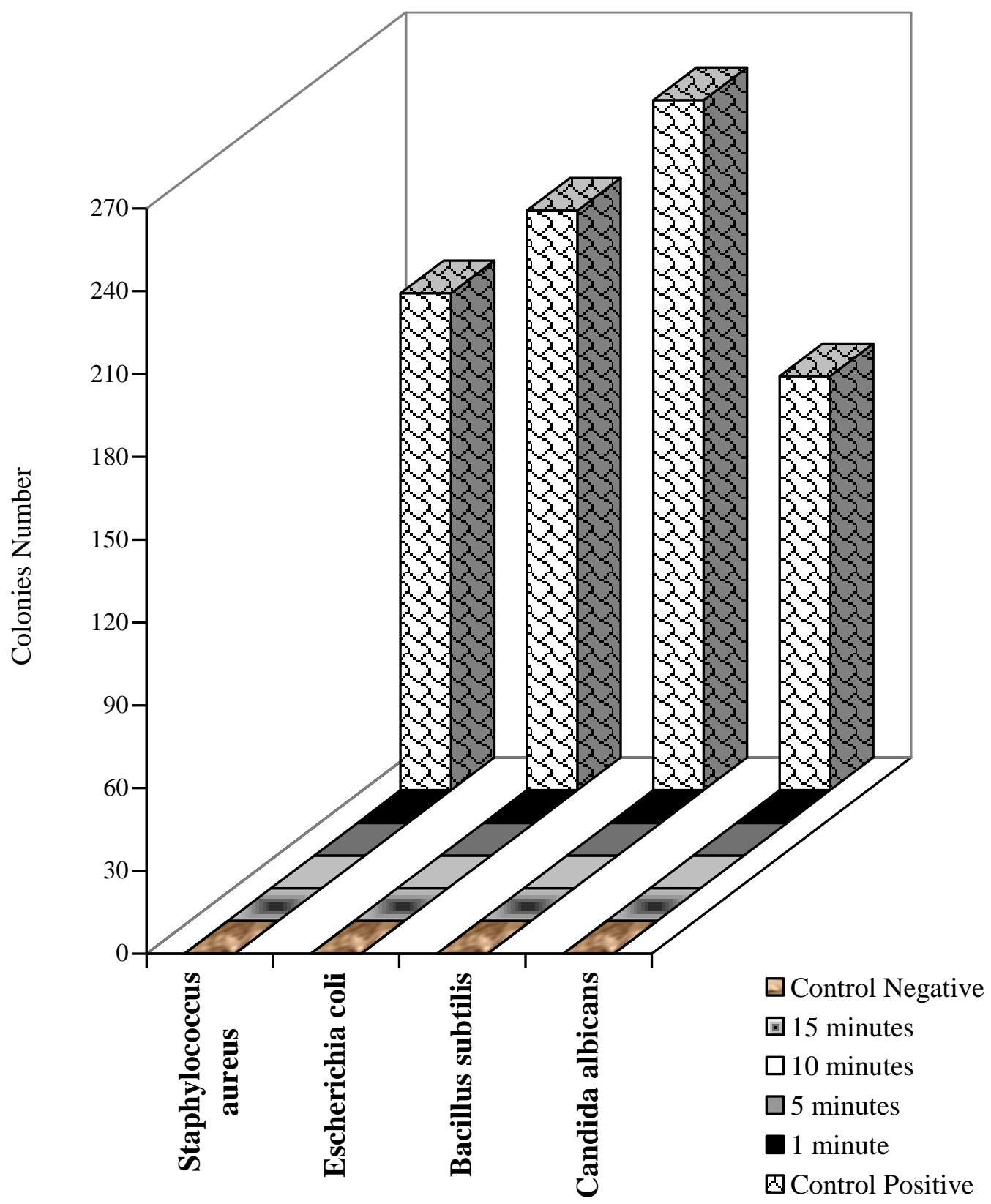

Figure 1 . The effect of $6 \%$ hydrogen peroxide in decontamination of gutta percha from different microorganisms at different periods 


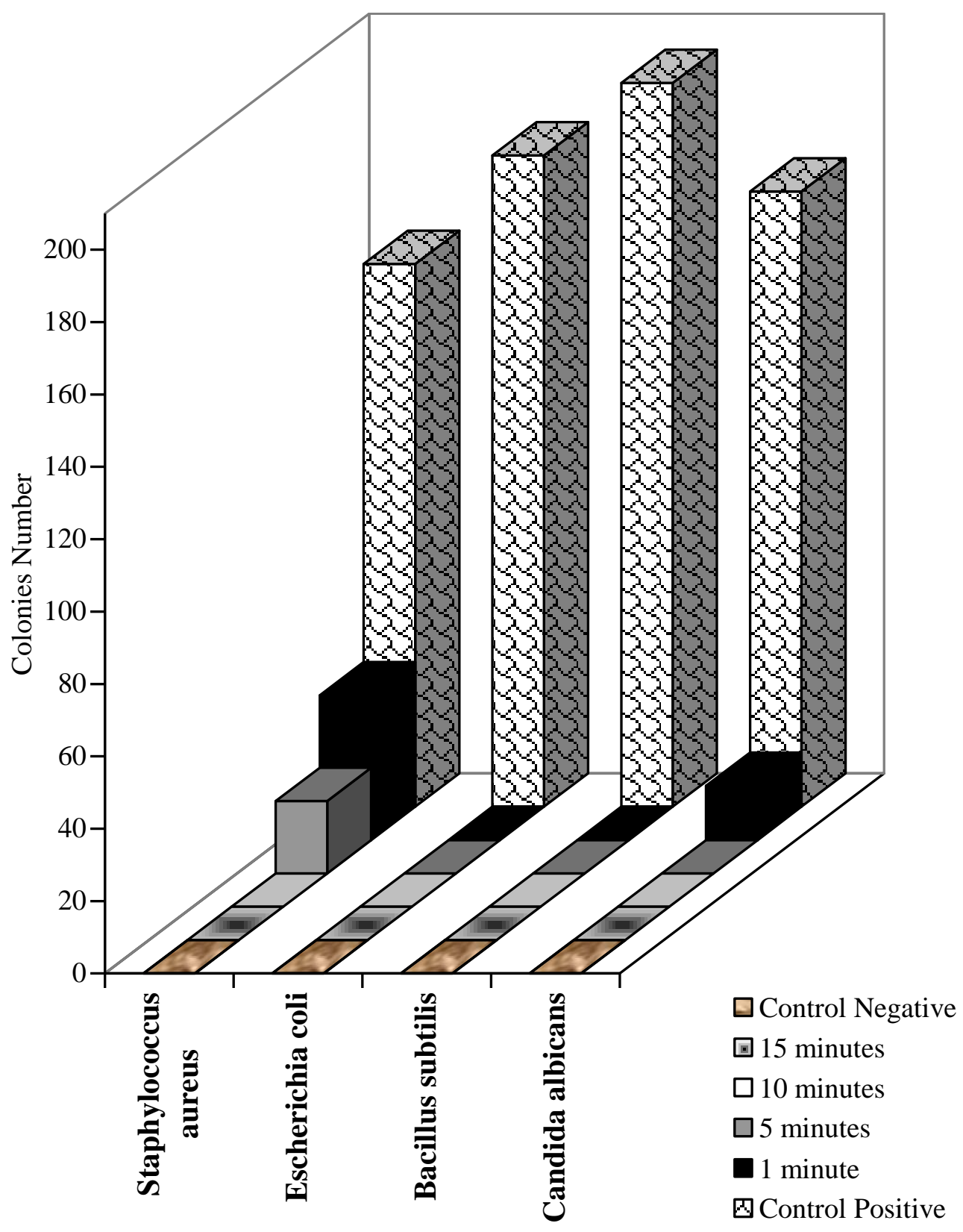

Figure 2. The effect of $2 \%$ Chlorhexidine in decontamination of gutta percha from different microorganisms at different periods 


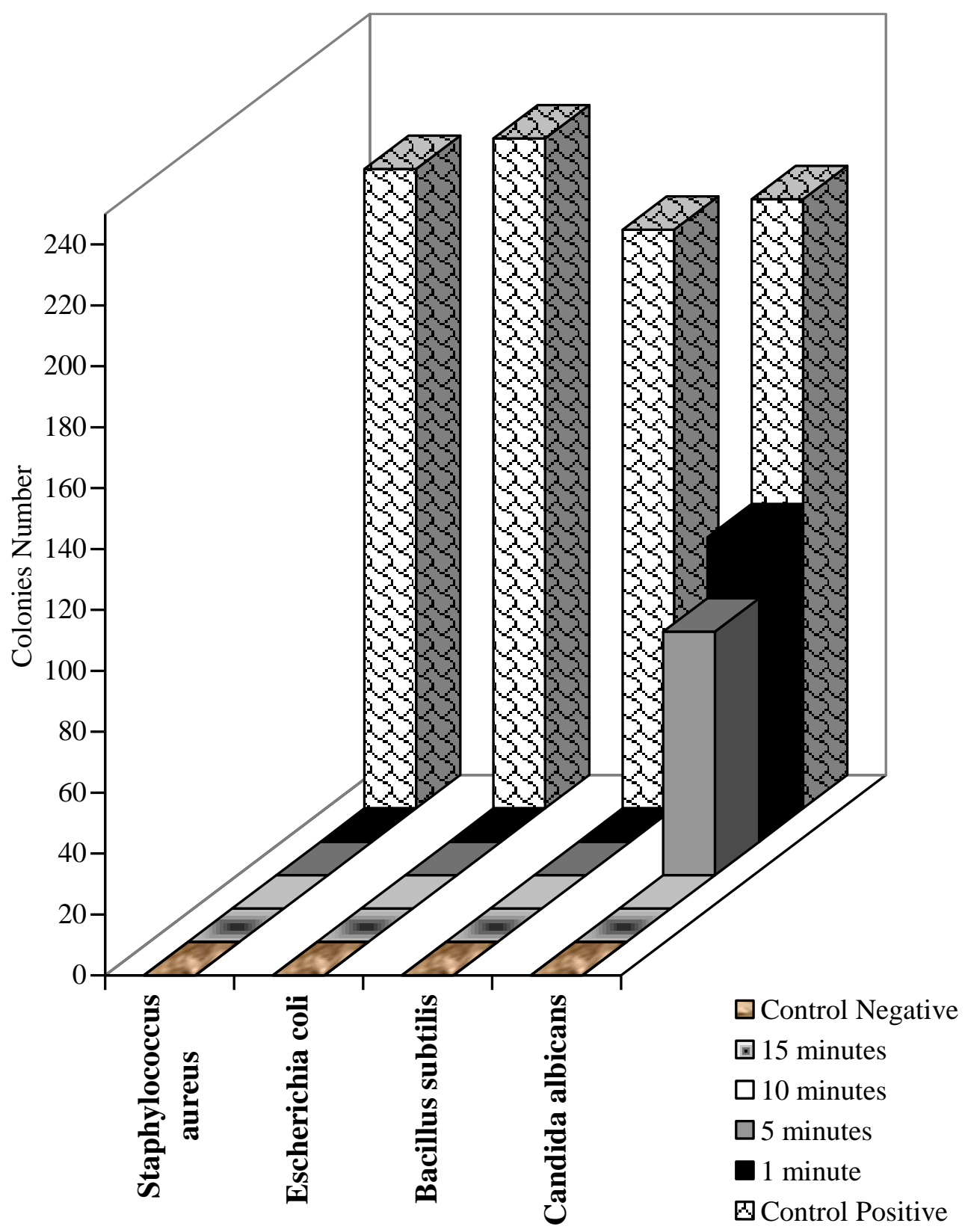

Figure 3. The effect of $1 \%$ Sodium Hypochlorite in decontamination of gutta percha from different microorganisms at different periods 


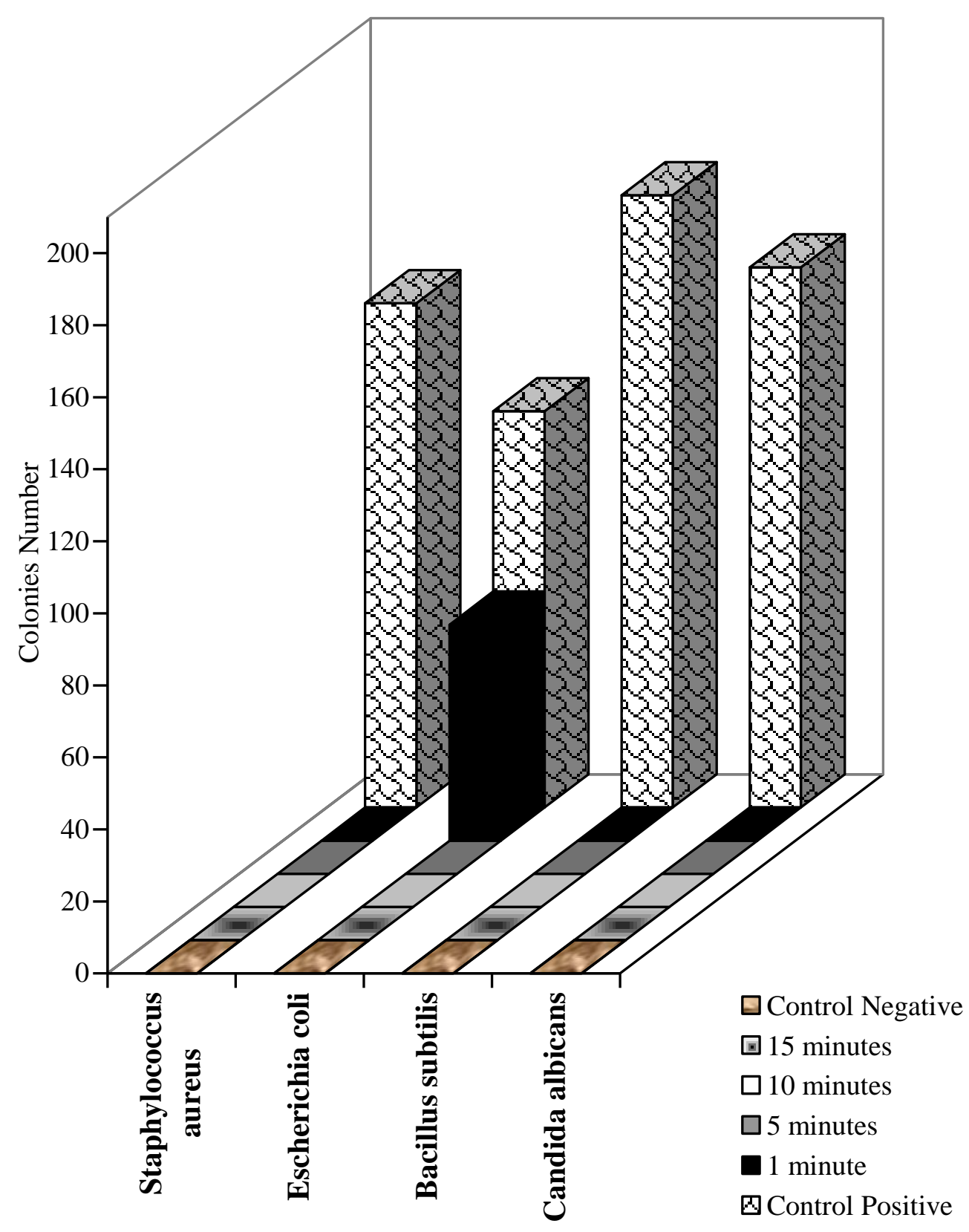

Figure 4. The effect of 70\% Ethyl Alcohol in decontamination of gutta percha from different microorganisms at different periods

\section{DISCUSSION}

A major objective of endodontic therapy is the establishment of a root canal system free of contaminants, especially microorganisms. Considerable effort to remove microorganisms already present in the root canal system ${ }^{(9)}$. However, in contrast to the care that is taken in cleaning the can- als, obturation is often accomplished using gutta percha cones directly from their storage container without regard to their sterility. Since most manufactures do not claim that their cones are sterile, this practice could reduce the achieving of a successful result ${ }^{(10)}$. Although most gutta perch cones appear to be initially sterile, 
there is a risk of contamination from both air borne and physical sources during their storage $^{(11)}$.

The selection of chemical agents for decontamination of gutta percha cones in the present study was based on literature data $(5,10,11,12,13)$. Among the chemical agents tested, hydrogen peroxide at $6 \%$ which was the most efficient product. It eliminated bacterial cells and fungi in 1 minute. Pennanchin and Alvers ${ }^{(12)}$ investigated the efficiency of hydrogen peroxide in the decontamination of gutta percha cones. Cones were inoculated with saliva, purulent materials obtained from extracted teeth or from patients with apical abscesses. Hydrogen peroxide at $6 \%$ was effective in the rapid decontamination of cones in 10 minutes. Such differences probably due to the effect of pus or saliva on the efficiency of hydrogen peroxide. While the results of the study of Cardoso et al. ${ }^{(11)}$ was comparable with the present study concerning hydrogen peroxide on the same microorganisms.

The use of $2 \%$ chlorhexidine in the present study, showed ability to decontaminate gutta percha cones after $10 \mathrm{mi}-$ nutes. Stablholz et al. ${ }^{(13)}$ showed that $1.5 \%$ chlorhexidine was effective within 30 second for disinfection of cones from Staphylococcus aureus, Streptococcus, Bacillus subtilis, and Candida albicans. In another study the efficiency of $2 \%$ chlorhexidine to the gutta percha cones decontamination after 10 minutes was evidenced ${ }^{(6)}$. Such differences between different studies is probably due to several factors such as differences in the concentration of chemical agentand strain of microorganisms.

The use of $1 \%$ of sodium hypochlorite in this study showed that it was effective after 10 minutes. While several studies confirmed the efficiency of 5.25\%, 5\%, $4.5 \%$, and $2.5 \%$ of sodium hypochlorite, there were few studies of the effect of $1 \%$ sodium hypochlorite ${ }^{(4,7,10,14,15)}$. Gomes et $a l .{ }^{(16)}$ evaluated the effectiveness of $5.25 \%$ sodium hypochlorite at in disinfecting of gutta percha cones from Bacillus subtilis. Results showed that sodium hypochlorite eliminated spores from gutta percha in 1 minute which is comparable with the present result. Ozalp et al. ${ }^{(17)}$ studied the effectiveness of a lower concentration of sodium hypochlorite $(2.5 \%)$ for rapid sterilization of gutta percha cones contaminated with Bacillus subtilis and found similar results. Pang et al. ${ }^{(18)}$ found similar effect on Staphylococcus spp.

With regard to the effectiveness of $70 \%$ of ethyl alcohol in rapid gutta percha cones decontamination. The present study showed that its effect after 5 minutes. Stabholz et $a l .{ }^{(13)}$ and Cardoso et $a .^{(12)}$ showed that bactericidal activity of $70 \%$ ethyl alcohol occured in 1 minute, and this agent was unable to destroy Bacillus subtilis even after 15 minute of contact. The differences in decontamination effect of present study from other studies may have been related to several factors such as experimental methods, concentration and type of chemical agent, characteristic of the microorganisms, and time of analysis or exposure.

\section{CONCLUSIONS}

The four chemical agents were effective for rapid decontamination of gutta perch cones, as hydrogen peroxide requiring shorter period of use about 1 minute, sodium hypochlorite and chlorhexidine were effective after 10 minutes, and finally ethyl alcohol was proved to be effective after 5 minutes.

\section{REFERENCES}

1. Siqueira JF, Rjcas IN, Santos SR, Lima KA Magalhaes FA, Uzeda M. E. Efficacy of instrumentation and irrigation regimes in reducing the bacterial population within root canal. $J$ Endod. 2002;28:181-185.

2. Attin T, Zirkel C, Peis K. Antibacterial propertiesof electron beam sterilized gutta percha cones. $J$ Endod. 2001;27:172-177.

3. Leonarod MR. Evaluation of the sterility and antimicrobial activity of gutta percha cones. Braz Endod J.1997;2:51-54.

4. Hasheminya SM. Comparison of the effectiveness of three disinfectant solutions in disinfection of gutta percha cones in one minute. J Dent. 2006;18:4-10.

5. Souza RE, Souza EA, Sousa-Neto MD, Pietro RC. In vitro evaluation of different chemical agents for the decontami- 
nation of gutta percha cones. Pesiq Odont Braz. 2003;17:20-25.

6. Cardoso CL, Redmerski R, Garcia LB, Hidaigo MM. Rapid decontamination of gutta-percha cones with alcohol iodine. Acta Scientiarum. 2001; 23:719-724.

7. Valois CRA, Silva LP, Azevedo RB. Effects of 2\% chlorhexidine and 5.25\% sodium hypochlorite on gutta percha cones studied by atomic force microscopy. Int Endod J. 2005; 38:425-429.

8. Motta D, Figueiredo PG, Maltos CBO, Nicoli SMM, Carvalhais HPM. Efficacy of chemical sterilization and storage conditions of gutta percha cones. Int Endod J. 2001;34:435-439.

9. Higgin HM, Newton CW, Charles JP. The use of paraformaldehyde powder for the sterile storage of gutta percha cones. J Endod. 1987;12:242-248.

10. Gomes BF, Rubel RL, Fried I. Decontamination of gutta perch cones by different concentrations of sodium hypochlorite. Rev Assoc Paul Cir Dent. 2001;55:27-31.

11. Cardoso CL, Redmerski R, Bettencourt NLR, Kotaka CR. Effectiveness of different chemical agents in rapid decontamination of gutta-percha cones. Braz J Microb. 2000; 31:67-71.

12. Pennanchin R, Alvares S. Decontamination of gutta percha cones by ethyl alco- hol. Rev Poul Endod.1981;2:4-8.

13. Stabholz A, Friedman S, Helling I, Sela MN. Efficiency of different chemical agents in decontamination of gutta percha cones. Int Endod J. 1987;20:211216.

14. Doolittle TR, Rubel RL, Fried I. The effectiveness of common office disinfection procedure for gutta percha and silver points. $N \quad Y$ State Dent $J$. 1995;41:409-414.

15. Kotaka CR, Redmersk R, Quieiroz AF, Cardoso CL. Rapid decontamination of gutta percha cones in endodontic practice. Rev Fac Odentol Bauru. 1998;6:7380.

16. Gomes BP, Vianna ME, Matsumoto CU, Rossi VD, Zaia AA, Filho FJ. Disinfection of gutta percha cones with chlorhexidine and sodium hypochlorite. Oral Surg Oral Med Oral Pathol Oral Radiol Endod. 2005;100:512-515.

17. Ozalp N, Okte Z, Ozcelik B. The rapid sterilization of gutta percha cones with sodium hypochlorite and glutaraldehyde. J Endod. 2006;32:1202-1204.

18. Pang NS, Jung Y, Bae KS, Baek SH. Effects of short term chemical disinfection of gutta percha cones: Identification of affected microbes and alteration in surface texture and physical properties. J Endod. 2007;33:594-598. 